\title{
The Physics of Polarization
}

\section{Egidio Landi Degl'Innocenti}

\author{
Department of Physics and Astronomy, University of Firenze, Italy \\ email: landie@arcetri.astro.it
}

\begin{abstract}
The introductory lecture that has been delivered at this Symposium is a condensed version of an extended course held by the author at the XII Canary Island Winter School from November 13 to November 21, 2000. The full series of lectures can be found in Landi Degl'Innocenti (2002). The original reference is organized in 20 Sections that are here itemized: 1. Introduction, 2. Description of polarized radiation, 3. Polarization and optical devices: Jones calculus and Muller matrices, 4. The Fresnel equations, 5. Dichroism and anomalous dispersion, 6. Polarization in everyday life, 7. Polarization due to radiating charges, 8. The linear antenna, 9. Thomson scattering, 10. Rayleigh scattering, 11. A digression on Mie scattering, 12. Bremsstrahlung radiation, 13. Cyclotron radiation, 14. Synchrotron radiation, 15. Polarization in spectral lines, 16. Density matrix and atomic polarization, 17. Radiative transfer and statistical equilibrium equations, 18. The amplification condition in polarized radiative transfer, and 19. Coupling radiative transfer and statistical equilibrium equations.

The introductory lecture delivered at the Symposium has covered the subjects itemized above with the exclusion of Sections 10, 11, 14, 18, and 19.
\end{abstract}

Keywords. Polarization, radiative transfer, scattering, stars: atmospheres, Sun: chromosphere

\section{References}

Landi Degl'Innocenti, E. 2002, in: J. Trujillo Bueno, F. Moreno Insertis \& F. Sánchez (eds.), Astrophysical Spectropolarimetry, Cambridge Univesity Press, pp. 1-53 